\title{
Near-best univariate spline discrete quasi-interpolants on non-uniform partitions
}

\author{
D. Barrera, M.J. Ibañez, P. Sablonnière, D. Sbibih
}

\begin{abstract}
Univariate spline discrete quasi-interpolants (abbr. dQIs) are approximation operators using B-spline expansions with coefficients which are linear combinations of discrete values of the function to be approximated. When working with nonuniform partitions, the main challenge is to find dQIs which have both good approximation orders and bounded uniform norms independent of the given partition. Near-best dQIs are obtained by minimizing an upper bound of the infinite norm of dQIs depending on a certain number of free parameters, thus reducing this norm. This paper is devoted to the study of some families of near-best dQIs of approximation order 2 .
\end{abstract}

\section{$\S 1 . I n t r o d u c t i o n$}

A spline quasi-interpolant (abbr. QI) of $f$ has the general form

$$
Q f=\sum_{\alpha \in A} \mu_{\alpha}(f) B_{\alpha}
$$

where $\left\{B_{\alpha}, \alpha \in A\right\}$ is a family of B-splines forming a partition of unity and $\left\{\mu_{\alpha}(f), \alpha \in A\right\}$ is a family of linear functionals which are local in the sense that they only use values of $f$ in some neighbourhood of $\Sigma_{\alpha}=\operatorname{supp}\left(B_{\alpha}\right)$. The main interest of QIs is that they provide good approximants of functions without solving any linear system of equations. In the literature, one can find the three following types of QIs:

(i) Differential QIS (abbr. DQIs) : the linear functionals are linear combinations of values of derivatives of $f$ at some point in $\Sigma_{\alpha}$ (see e.g. [5-7]).

(ii) Discrete QIs (abbr. dQIs) : the linear functionals are linear combinations of values of $f$ at some points in some neighbourhood of $\Sigma_{\alpha}$ (see e.g. [1-3], [6], [9], [11], [13], [15-16], [24]).

(iii) Integral QIs (abbr. iQIs) : the linear functionals are linear combinations of weighted mean values of $f$ in some neighbourhood of $\Sigma_{\alpha}$ (see e.g. [2-3], [6], [13-14], [24-25]). 
In this paper and a subsequent one, we shall study various types of univaraite dQIs and iQIs, more specifically those that we call near-best QIs which are defined as follows:

(dQIs) assume that $\mu_{\alpha}(f)=\sum_{\beta \in F_{\alpha}} \lambda_{\alpha}(\beta) f\left(x_{\beta}\right)$ where the finite set of points $\left\{x_{\beta}, \beta \in F_{\alpha}\right\}$ lies in some neighbourhood of $\Sigma_{\alpha}$. Then it is clear that, for $\|f\|_{\infty} \leq 1$ and $\alpha \in A,\left|\mu_{\alpha}(f)\right| \leq\left\|\lambda_{\alpha}\right\|_{1}$, where $\lambda_{\alpha}$ is the vector with components $\lambda_{\alpha}(\beta)$, from which we deduce immediately

$$
\|Q\|_{\infty} \leq \sum_{\alpha \in A}\left|\mu_{\alpha}(f)\right| B_{\alpha} \leq \max _{\alpha \in A}\left|\mu_{\alpha}(f)\right| \leq \max _{\alpha \in A}\left\|\lambda_{\alpha}\right\|_{1}=\nu_{1}(Q) .
$$

Now, assuming that $n=\operatorname{card}\left(F_{\alpha}\right)$ for all $\alpha$, we can try to find a $\lambda_{\alpha}^{*} \in \mathbb{R}^{n}$ solution of the minimization problem

$$
\left\|\lambda_{\alpha}^{*}\right\|_{1}=\min \left\{\left\|\lambda_{\alpha}\right\|_{1} ; \lambda_{\alpha} \in \mathbb{R}^{n}, V_{\alpha} \lambda_{\alpha}=b_{\alpha}\right\}
$$

where the linear constraints express that $Q$ is exact on some subspace of polynomials. Thus, we finally obtain

$$
\|Q\|_{\infty} \leq \nu_{1}^{*}(Q)=\max _{\alpha \in A}\left\|\lambda_{\alpha}\right\|_{1}
$$

(iQIs) assume that $\mu_{\alpha}(f)=\sum_{\beta \in F_{\alpha}} \lambda_{\alpha}(\beta) \int_{\Sigma_{\beta}} M_{\beta}(t) f(t) d t$, where the Bsplines $M_{\beta}$ are normalized by $\int M_{\beta}=1$. Once again, for $\|f\|_{\infty} \leq 1$, we have

$$
\left|\mu_{\alpha}(f)\right| \leq \sum_{\beta \in F_{\alpha}}\left|\lambda_{\alpha}(\beta)\left\|\int_{\Sigma_{\beta}} M_{\beta}(t) f(t) d t\left|\leq \sum_{\beta \in F_{\alpha}}\right| \lambda_{\alpha}(\beta) \mid=\right\| \lambda_{\alpha} \|_{1}\right.
$$

whence, as we obtained above for dQIs,

$$
\|Q\|_{\infty} \leq \max _{\alpha \in A}\left\|\lambda_{\alpha}\right\|_{1}=\nu_{1}^{*}(Q)
$$

As emphasized by de Boor (see e.g. [5], chapter XII), a QI defined on non uniform partitions has to be uniformly bounded independently of the partition (abbr. UB) in order to be interesting for applications. Therefore, the aim of this paper is to define some families of dQIs satisfying this property and having the smallest possible norm. As in general it is difficult to minimize the true norm of the operator, we have chosen to solve the minimization problems defined above. A further paper [25] will develop the case of iQIs on nonuniform partitions. A few results are given in [24].

The paper extends some results of [1][13] and is organized as follows. We first recall some "classical" QIs of various types and we verify that they are UB. Then we define and study several families of discrete and integral 
QIs, depending on a finite number of parameters, for which we can find $\nu_{1}^{*}(Q)$. We show that this problem has always a solution (in general non unique). We give more specific examples for quadratic and cubic splines. Of particular interest are the results of theorems 3,5 and 6 where we show that some families of dQIs are uniformly bounded independently of the partition. Finally, we briefly give some applications to the approximation of functions, to quadrature formulas and to pseudo-spectral methods (see e.g. [12], [29]). A parallel study of spline QIs is done in [2] for uniform partitions of the real line and in [3] for some uniform triangulations of the plane.

\section{$\S 2$. Notations}

We shall use classical B-splines of degree $m$ on a bounded interval $I=[a, b]$ or on $I=\mathbb{R}$. For the sake of simplicity, in the case $I=\mathbb{R}$, we take an increasing sequence of knots $T=\left\{t_{i}, i \in \mathbb{Z}\right\}$. In the case $I=[a, b]$, we take the usual sequence $T$ of knots defined by (see [5][11][19][28]):

$$
\begin{gathered}
a=t_{-m}=\ldots=t_{0}, \quad b=t_{n}=\ldots=t_{n+m} \\
a<t_{1}<t_{2}<\ldots<t_{n-1}<b
\end{gathered}
$$

For $J=\{0, \ldots, n+m-1\}$, the family of B-splines $\left\{B_{j}, j \in J\right\}$, with support $\Sigma_{j}=\left[t_{j-m}, t_{j+1}\right]$ is a basis of the space $S_{m}(I, T)$ of splines of degree $m$ on the interval $I$ endowed with the partition $T$. These B-splines form a partition of unity, i.e. $\sum_{j \in J} B_{j}=1$. We denote $h_{i}=t_{i}-t_{i-1}$ for all indices $i$.

Let $\mathbb{N}_{m}=\mathbb{N} \cap[0, m-1]$ and $T_{j}=\left\{t_{j-r}, r \in \mathbb{N}_{m}\right\}$ : we recall that the elementary symmetric functions $\sigma_{l}(T)$ of the $m$ variables in $T_{j}$ are defined by $\sigma_{0}\left(T_{j}\right)=0$ and for $1 \leq l \leq m$, by

$$
\sigma_{l}\left(T_{j}\right)=\sum_{0 \leq r_{1}<r_{2}<\ldots<r_{l} \leq m-1} t_{j-r_{1}} t_{j-r_{2}} \ldots t_{j-r_{l}} .
$$

Let $C_{m}^{l}=\frac{m !}{l !(m-l) !}$ be the binomial coefficients, then the monomials $e_{l}(x)=$ $x^{l}$ can be written $e_{l}=\sum_{i \in J} \theta_{i}^{(l)} B_{i}$, with $\theta_{i}^{(l)}=\sigma_{l}\left(T_{i}\right) / C_{m}^{l}$, for $0 \leq l \leq m$. This is a direct consequence of Marsden's identity ([3], chapter IX).

*************************************

\section{$\S 3$. Differential QIs}

For all $j \in J$, we define $\psi_{j}(t)=\prod_{r \in \mathbf{N}_{m}}\left(t_{j-r}-t\right.$ ) (thus $\psi_{j} \in \mathbb{P}_{m}$ for all $j \in J$ ). From de Boor and Fix [7] or de Boor ([5], chapter IX), we know that for any $\tau \in \Sigma_{j}$, the functionals

$$
\lambda_{j}(f)=\frac{1}{m !} \sum_{l=0}^{m}(-1)^{m-l} D^{m-l} \psi_{j}(\tau) D^{l} f(\tau)
$$


are dual functionals of B-splines, i.e. they satisfy, for all pairs $(i, j) \in J \times J$

$$
\lambda_{j}\left(B_{i}\right)=\delta_{i j}
$$

Therefore the differential quasi-interpolant (abbr. DQI)

$$
Q f=\sum_{j \in J} \lambda_{j}(f) B_{j}
$$

satisfies $Q B_{j}=B_{j}$ for all $j \in J$, i.e. $Q$ is a projector on the space $S_{m}(I, T)$. In practice, it is interesting to choose $\tau=\theta_{j}=\frac{1}{m} \sum_{s \in \mathbb{N}_{m}} t_{j-s}=$ $s_{1}\left(T_{j}\right)=\theta_{j}^{(1)}$. However, the computation of $\lambda_{j}(f)$ needs the evaluation all derivatives of polynomials $\psi_{j}$. Another method consists in writing $Q$ in the form

$$
Q f=\sum_{i \in J} \tilde{\lambda}_{i}(f) B_{i}
$$

whose coefficient functionals are defined by

$$
\tilde{\lambda}_{i}(f)=\sum_{l=0}^{m} a_{l}\left(\theta_{i}\right) \frac{D^{l} f\left(\theta_{i}\right)}{l !},
$$

and to impose that $Q$ be exact on monomials of degree at most $m$

$$
Q e_{k}=e_{k} \text { for } 0 \leq k \leq m .
$$

Setting $\alpha_{l}\left(\theta_{i}\right)=\theta_{i}^{-l} a_{l}\left(\theta_{i}\right)$ and $\beta_{s}\left(\theta_{i}\right)=\theta_{i}^{-s} \theta_{i}^{(s)}$, we obtain the following system of linear equations, for $0 \leq s \leq m$ :

$$
\sum_{l=0}^{s} C_{s}^{l} \alpha_{l}\left(\theta_{i}\right)=\beta_{s}\left(\theta_{i}\right)
$$

The solutions of this system are given by

$$
\alpha_{s}\left(\theta_{i}\right)=\sum_{l=0}^{s}(-1)^{s-l} C_{s}^{l} \beta_{l}\left(\theta_{i}\right)
$$

Thus we finally obtain

Theorem 1. The coefficients of the differential forms $\left\{\tilde{\lambda}_{i}(f), i \in J\right\}$, are given, for $0 \leq s \leq m$, by

$$
a_{s}\left(\theta_{i}\right)=\sum_{l=0}^{s}(-1)^{s-l} C_{s}^{l} \theta_{i}^{s-l} \theta_{i}^{(l)}
$$


However, these DQIs need the values of derivatives of $f$, so they are not very easy to use in applications and we will not study them any more. Let us only give examples of quadratic and cubic DQIs.

Example 1: Quadratic spline DQIs (see also section 4 below). In de Boor's form, we have for $\tau=\theta_{j}: \lambda_{j}(f)=f\left(\theta_{j}\right)-\frac{1}{2}\left(\theta_{j}^{2}-\theta_{j}^{(2)}\right) D^{2} f\left(\theta_{j}\right)$, where $\theta_{j}=\frac{1}{2}\left(t_{j-1}+t_{j}\right)$ and $\theta_{j}^{(2)}=t_{j-1} t_{j}$, whence $\theta_{j}^{2}-\theta_{j}^{(2)}=\frac{1}{4}\left(t_{j-1}-\right.$ $\left.t_{j}\right)^{2}$ and finally $\lambda_{j}(f)=f\left(\theta_{j}\right)-\frac{1}{8} h_{i}^{2} D^{2} f\left(\theta_{j}\right)$. Theorem 1 gives $a_{0}\left(\theta_{j}\right)=$ $1, a_{1}\left(\theta_{j}\right)=0, a_{2}\left(\theta_{j}\right)=\theta_{j}^{2}-\theta_{j}^{(2)}$, hence $\tilde{\lambda}_{j}(f)=\lambda_{j}(f)$.

Example 2: Cubic spline DQIs. In de Boor's form, we have for $\tau=\theta_{j}$ : $\lambda_{j}(f)=f\left(\theta_{j}\right)-\frac{1}{2}\left(\theta_{j}^{2}-\theta_{j}^{(2)}\right) D^{2} f\left(\theta_{j}\right)-\frac{1}{6} \psi_{j}\left(\theta_{j}\right) D^{3} f\left(\theta_{j}\right)$, with $\theta_{j}=\frac{1}{3}\left(t_{j-2}+\right.$ $\left.t_{j-1}+t_{j}\right)$ and $\theta_{j}^{(2)}=\frac{1}{3}\left(t_{j-2} t_{j-1}+t_{j-1} t_{j}+t_{j-2} t_{j}\right)$, whence $\theta_{j}^{2}-\theta_{j}^{(2)}=$ $\frac{1}{9}\left(h_{i-1}^{2}+h_{i-1} h_{i}+h_{i}^{2}\right)$ and $\psi_{j}\left(\theta_{j}\right)=\left(t_{j}-\theta_{j}\right)\left(t_{j-1}-\theta_{j}\right)\left(t_{j-2}-\theta_{j}\right)$. Finally, we obtain $\lambda_{j}(f)=f\left(\theta_{j}\right)-\frac{1}{18}\left(h_{i-1}^{2}+h_{i-1} h_{i}+h_{i}^{2}\right) D^{2} f\left(\theta_{j}\right)-\frac{1}{162}\left(2 h_{i-1}+\right.$ $\left.h_{i}\right)\left(h_{i}-h_{i-1}\right)\left(h_{i-1}+2 h_{i}\right) D^{3} f\left(\theta_{j}\right)$. Theorem 1 gives $a_{0}\left(\theta_{j}\right)=1, a_{1}\left(\theta_{j}\right)=$ $0, a_{2}\left(\theta_{j}\right)=\theta_{j}^{2}-\theta_{j}^{(2)}, a_{3}\left(\theta_{j}\right)=\theta_{j}^{(3)}-3 \theta_{j} \theta^{(2)}+2 \theta_{j}^{3}=\frac{1}{27}\left(2 h_{i-1}+h_{i}\right)\left(h_{i}-\right.$ $\left.h_{i-1}\right)\left(h_{i-1}+2 h_{i}\right)$, whence $\tilde{\lambda}_{j}(f)=\lambda_{j}(f)$.

\section{§4. Uniformly bounded discrete QIs exact on $\mathbb{P}_{2}$}

It is now possible to derive discrete QIs from the preceding DQIs by replacing the values of derivatives $D^{l} f\left(\theta_{i}\right) / l$ ! of $f$ by divided differences at the points $\theta_{r}$ lying in $\Sigma_{i}$. Doing this, we loose the property of projection on $S_{m}(I, T)$. However, by choosing conveniently the divided differences, we can obtain some families of dQIs which are UB and exact on specific subspaces of polynomials.

Let us construct for example a family of dQIs of degree $m$ which are exact on $\mathbb{P}_{2}$. We start from functionals which are truncations at order 2 of those of the preceding section:

$$
\lambda_{j}^{(2)}(f)=\frac{1}{m !} \sum_{l=0}^{2}(-1)^{m-l} D^{m-l} \psi_{j}(\tau) D^{l} f(\tau) .
$$

As $\psi_{j}(t)$ is of degree $m$, we obtain successively $D^{m} \psi_{j}(\tau)=(-1)^{m} m$ !,

$D^{m-1} \psi_{j}(\tau)=(-1)^{m} m !\left(\tau-\theta_{j}\right), D^{m-2} \psi_{j}(\tau)=\frac{1}{2}(-1)^{m} m !\left(\tau^{2}-2 \theta_{j} t+\theta_{j}^{(2)}\right)$. More specifically, taking $\tau=\theta_{j}$, we get

$$
D^{m-1} \psi_{j}\left(\theta_{j}\right)=0, \quad D^{m-2} \psi_{j}\left(\theta_{j}\right)=\frac{1}{2}(-1)^{m} m !\left(\theta_{j}^{(2)}-\theta_{j}^{2}\right)
$$

and we obtain the DQI exact on $\mathbb{P}_{2}$

$$
Q_{2} f=\sum_{j \in J} \lambda_{j}^{(2)}(f) B_{j}
$$


whose coefficient functionals are given by

$$
\lambda_{j}^{(2)}(f)=f\left(\theta_{j}\right)-\frac{1}{2}\left(\theta_{j}^{2}-\theta_{j}^{(2)}\right) D^{2} f\left(\theta_{j}\right)
$$

We recall the expansion (se e.g. [9][17]):

$$
\bar{\theta}_{j}^{(2)}=\theta_{j}^{2}-\theta_{j}^{(2)}=\frac{1}{m^{2}(m-1)} \sum_{(r, s) \in \mathbb{N}_{m}^{2}, r<s}\left(t_{j-r}-t_{j-s}\right)^{2}>0 .
$$

On the other hand, $\frac{1}{2} D^{2} f\left(\theta_{j}\right)$ coincide on the space $\mathbb{P}_{2}$ with the second order divided difference $\left[\theta_{j-1}, \theta_{j}, \theta_{j+1}\right] f$, therefore the dQI defined by

$$
Q_{2}^{*} f=\sum_{j \in J} \mu_{j}^{(2)}(f) B_{j}
$$

with coefficient functionals

$$
\mu_{j}^{(2)}(f)=f\left(\theta_{j}\right)-\bar{\theta}_{j}^{(2)}\left[\theta_{j-1}, \theta_{j}, \theta_{j+1}\right] f,
$$

is also exact on $\mathbb{P}_{2}$. Moreover, one can write

$$
\mu_{i}^{(2)}(f)=a_{i} f_{i-1}+b_{i} f_{i}+c_{i} f_{i+1}
$$

with $a_{i}=-\bar{\theta}_{i}^{(2)} / \Delta \theta_{i-1}\left(\Delta \theta_{i-1}+\Delta \theta_{i}\right), \quad b_{i}=1+\bar{\theta}_{i}^{(2)} / \Delta \theta_{i-1} \Delta \theta_{i}$, $c_{i}=-\bar{\theta}_{i}^{(2)} / \Delta \theta_{i}\left(\Delta \theta_{i-1}+\Delta \theta_{i}\right)$. So, according to the introduction

$$
\left\|Q_{2}^{*}\right\|_{\infty} \leq \max _{i \in J}\left(\left|a_{i}\right|+\left|b_{i}\right|+\left|c_{i}\right|\right) \leq 1+2 \max _{i \in J} \bar{\theta}_{i}^{(2)} / \Delta \theta_{i-1} \Delta \theta_{i} .
$$

The following theorem extends a result given for quadratic splines in $[13][22][23]$.

Theorem 2. For any degree $m$, the $d Q I_{S} Q_{2}^{*}$ are uniformly bounded. More specifically, for all partitions of $I$ :

$$
\left\|Q_{2}^{*}\right\|_{\infty} \leq\left[\frac{1}{2}(m+4)\right]
$$

Proof: We only give the proof for $m=2 k+1$, the case $m=2 k$ being similar. For the sake of simplicity, we take $j=k$, i.e. we shall determine an upper bound of the ratio

$$
N_{k} / D_{k}=\bar{\theta}_{k}^{(2)} / \Delta \theta_{k-1} \Delta \theta_{k}
$$


with

$$
N_{k}=\bar{\theta}_{k}^{(2)}=\frac{1}{m^{2}(m-1)} \sum_{1 \leq r<s \leq m}\left(t_{r}-t_{s}\right)^{2} .
$$

Setting $H=\sum_{i=1}^{m-1} h_{i}$, then we get a lower bound for the denominator

$$
D_{k}=\frac{1}{m^{2}}\left(t_{m-1}-t_{0}\right)\left(t_{m}-t_{1}\right)=\frac{1}{m^{2}}\left(h_{0}+H\right)\left(H+h_{m-1}\right) \geq \frac{H^{2}}{m^{2}}
$$

The numerator $N_{k}$ is composed of $k-1$ pairs of sums $\left(S_{p}, S_{p}^{\prime}\right)$

$$
S_{p}=\sum_{s-r=p}\left(t_{r}-t_{s}\right)^{2}, \quad S_{p}^{\prime}=\sum_{s-r=k+p-1}\left(t_{r}-t_{s}\right)^{2}
$$

for $1 \leq p \leq k-1$. Both sums contain at most $p$ times the terms $h_{i}^{2}$ and $2 h_{i} h_{j}(i \neq j)$, hence we can write $S_{p}+S_{p}^{\prime} \leq 2 p H^{2}$, which implies

$$
N_{k} \leq \frac{2 H^{2}}{(m-1)^{2}(m-2)}(1+2+\ldots k-1)=\frac{k S^{2}}{2(m-1)^{2}}
$$

so, we get

$$
N_{k} / D_{k} \leq k / 2
$$

and finally, for $m=2 k+1$ odd

$$
\left\|Q_{2}^{*}\right\|_{\infty} \leq k+2=\frac{1}{2}(m+3)=\left[\frac{1}{2}(m+4)\right]
$$

For $m=2 k$, we obtain respectively $D_{k} \geq \frac{S^{2}}{4 k^{2}}$ and $N_{k} \leq \frac{S^{2}}{4(2 k-1)}$, whence $N_{k} / D_{k} \leq \frac{k^{2}}{2 k-1}$, and finally for $m=2 k$ even

$$
\left\|Q_{2}^{*}\right\| \leq k+2=\frac{1}{2}(m+4)=\left[\frac{1}{2}(m+4)\right]
$$

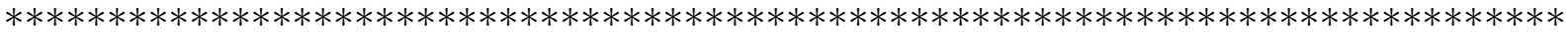
$* * * * * * * * * * * *$

\section{$\S 6$. Existence and characterization of near-best discrete QIs}




\subsection{Existence of near-best dQIs}

We consider the following family of dQIs defined, for the sake of simplicity, on $I=\mathbb{R}$ endowed with an arbitrary non-uniform increasing sequence of knots $T=\left\{t_{i} ; i \in \mathbb{Z}\right\}$,

$$
Q f=Q_{p, q} f=\sum_{i \in \mathbb{Z}} \mu_{i}(f) B_{i}
$$

Their coefficient functionals depend on $2 p+1$ parameters, with $p \geq m$

$$
\mu_{i}(f)=\sum_{s=-p}^{p} \lambda_{i}(s) f\left(\theta_{i+s}\right)
$$

and they are exact on the space $\mathbb{P}_{q}$, where $q \leq \min (m, 2 p)$. The latter condition is equivalent to $Q e_{r}=e_{r}$ for all monomials of degrees $0 \leq r \leq q$. It implies that for all indices $i$, the parameters $\lambda_{i}(s)$ satisfy the system of $q+1$ linear equations:

$$
\sum_{s=-p}^{p} \lambda_{i}(s) \theta_{i+s}^{r}=\theta_{i}^{(r)}, \quad 0 \leq r \leq q
$$

The matrix $V_{i} \in \mathbb{R}^{(q+1) \times(2 p+1)}$ of this system, with coefficients $V_{i}(r, s)=$ $\theta_{i+s}^{r}$, is a Vandermonde matrix of maximal rank $q+1$, therefore there are $2 p-q$ free parameters. Denoting $b_{i} \in \mathbb{R}^{q+1}$ the vector in the right hand side, with components $b_{i}(r)=\theta_{i}^{(r)}, \quad 0 \leq r \leq q$, we consider the sequence of minimization problems, for $i \in \mathbb{Z}$ :

$$
\min \left\|\lambda_{i}\right\|_{1}, \quad V_{i} \lambda_{i}=b_{i}
$$

We have seen in the introduction that $\nu_{1}^{*}(Q)=\max _{i \in \mathbb{Z}} \min \left\|\lambda_{i}\right\|_{1}$ is an upper bound of $\left\|Q_{q}\right\|_{\infty}$ which is easier to evaluate than the true norm of the dQI.

Theorem 3. The above minimization problems have always solutions, which, in general, are non unique.

Proof: The objective function being convex and the domains being affine subspaces, these classical optimization problems have always solutions, in general non unique.

We postpone to sections 7 and 8 the computation of some optimal solutions in the case $q=2$. 


\subsection{Characterization of optimal solutions}

For $b \in \mathbb{R}^{m}$ and $A \in \mathbb{R}^{m \times n}$, let us consider the $l_{1}$-minimization problem

$$
\text { (1) } \min \|r(a)\|_{1}, \quad r(a)=b-A a \text {. }
$$

We recall the characterization of optimal solutions for $l_{1}$-problems given in [30], chapter 6. Define the sets

$$
\begin{gathered}
Z(a)=\left\{1 \leq i \leq m \mid r_{i}(a)=0\right\} \\
V(a)=\left\{v \in \mathbb{R}^{m} ;\|v\|_{\infty} \leq 1, v_{i}=\operatorname{sgn}\left(r_{i}(a)\right) \text { for } i \notin Z(a)\right\}
\end{gathered}
$$

Theorem 4. $a^{*}$ is a solution of (1) if and only if there exists a vector $v^{*} \in V\left(a^{*}\right)$ satisfying $A^{T} v^{*}=0$.

***********************************************************************

\section{$\S 7$. A general family of spline discrete QIs exact on $\mathbb{P}_{2}$}

In this section, we restrict our study to the subfamily of spline dQIs which are exact on $\mathbb{P}_{2}$, i.e; we choose $q=2$. We shall try to characterize optimal solutions in the sense of theorem 3 with the help of theorem 4 . Let

$$
Q_{p, 2} f=\sum_{i \in \mathbb{Z}} \mu_{i}(f) B_{i}
$$

where the coefficient functionals depend on $2 p+1$ parameters

$$
\mu_{i}(f)=\sum_{r=-p}^{p} \lambda_{i}(r) f\left(\theta_{i+r}\right)
$$

We shall need the following sets of indices

$$
\begin{gathered}
\bar{K}=\{-p, \ldots, p\}, \quad K^{*}=\{-p, 0, p\}, \quad K=\bar{K} \backslash K^{*} . \\
K=K_{1} \cup K_{2}, \quad K_{1}=\{-p+1, \ldots,-1\}, \quad K_{2}=\{1, \ldots, p-1\} .
\end{gathered}
$$

The three equations expressing the exactness of $Q_{p, 2}$ on $\mathbb{P}_{2}$ can be written

$$
\begin{gathered}
\lambda_{i}(-p)+\lambda_{i}(0)+\lambda_{i}(p)=1-\sum_{r \in K} \lambda_{i}(r) \\
\theta_{i-p} \lambda_{i}(-p)+\theta_{i} \lambda_{i}(0)+\theta_{i+p} \lambda_{i}(p)=\theta_{i}-\sum_{r \in K} \theta_{r} \lambda_{i}(r)
\end{gathered}
$$




$$
\theta_{i-p}^{2} \lambda_{i}(-p)+\theta_{i}^{2} \lambda_{i}(0)+\theta_{i+p}^{2} \lambda_{i}(p)=\theta_{i}^{(2)}-\sum_{r \in K} \theta_{r}^{2} \lambda_{i}(r)
$$

This system has a positive Vandermonde determinant

$$
V_{i}=V\left(\theta_{i-p}, \theta_{i}, \theta_{i+p}\right)=\left(\theta_{i}-\theta_{i-p}\right)\left(\theta_{i+p}-\theta_{i}\right)\left(\theta_{i+p}-\theta_{i-p}\right) .
$$

Let us denote by

$$
\left(\lambda_{i}^{*}(-p), \lambda_{i}^{*}(0), \lambda_{i}^{*}(p)\right)
$$

the unique solution of the above system with the right-hand side obtained by taking $\lambda_{i}(r)=0$ for all $r \in K$. Using Cramer's rule and the determinants $W_{i}(s)$ obtained by replacing the column of $\theta_{i+s}$ in $V_{i}$ by this rhs, we obtain

$$
\lambda_{i}^{*}(-p)=W_{i}(-p) / V_{i}, \quad \lambda_{i}^{*}(0)=W_{i}(0) / V_{i}, \quad \lambda_{i}^{*}(p)=W_{i}(p) / V_{i} .
$$

Then we can express the general solution of the above system in the form

$$
\begin{aligned}
\lambda_{i}(-p) & =\lambda_{i}^{*}(-p)-\sum_{r \in K_{1}} \alpha_{r} \lambda_{i}(r)+\sum_{s \in K_{2}} \alpha_{s} \lambda_{i}(s) \\
\lambda_{i}(0) & =\lambda_{i}^{*}(0)-\sum_{r \in K_{1}} \beta_{r} \lambda_{i}(r)-\sum_{s \in K_{2}} \beta_{s} \lambda_{i}(s) \\
\lambda_{i}(p) & =\lambda_{i}^{*}(p)+\sum_{r \in K_{1}} \gamma_{r} \lambda_{i}(r)-\sum_{s \in K_{2}} \gamma_{s} \lambda_{i}(s)
\end{aligned}
$$

The various coefficients are quotients of Vandermonde determinants

$$
\begin{aligned}
& \alpha_{r}=V\left(\theta_{r}, \theta_{i}, \theta_{i+p}\right) / V_{i}, \quad \alpha_{s}=V\left(\theta_{i}, \theta_{s}, \theta_{i+p}\right) / V_{i}, \\
& \beta_{r}=V\left(\theta_{i-p}, \theta_{r}, \theta_{i+p}\right) / V_{i}, \quad \beta_{s}=V\left(\theta_{i-p}, \theta_{s}, \theta_{i+p}\right) / V_{i}, \\
& \gamma_{r}=V\left(\theta_{i-p}, \theta_{r}, \theta_{i}\right) / V_{i}, \quad \gamma_{s}=V\left(\theta_{i-p}, \theta_{i}, \theta_{s}\right) / V_{i} .
\end{aligned}
$$

We denote by $Q_{p, 2}^{*}$ the spline dQI whose coefficient functionals are

$$
\mu_{i}^{*}(f)=\lambda_{i}^{*}(-p) f\left(\theta_{i-p}\right)+\lambda_{i}^{*}(0) f\left(\theta_{i}\right)+\lambda_{i}^{*}(p) f\left(\theta_{i+p}\right) .
$$

In that case, an upper bound of the norm is $\max _{i \in \mathbb{Z}} \nu_{i}^{*}$ where

$$
\nu_{i}^{*}=\left|\lambda_{i}^{*}(-p)\right|+\left|\lambda_{i}^{*}(0)\right|+\left|\lambda_{i}^{*}(p)\right|
$$

Theorem 5. For all $p \geq m=$ degree of the spline, the infinite norms of the spline dQIs $Q_{p, 2}^{*}$ are uniformly bounded by $\frac{m+1}{m-1}$. This bound is independent of $p$ and of the sequence of knots $T$.

Proof: We have to find a good upper bound of

$$
\nu_{i}^{*}=\left|\lambda_{i}^{*}(-p)\right|+\left|\lambda_{i}^{*}(0)\right|+\left|\lambda_{i}^{*}(p)\right|
$$


where, expanding the determinants, we have

$$
\begin{gathered}
\lambda_{i}^{*}(-p)=-\bar{\theta}_{i}^{(2)} /\left(\theta_{i+p}-\theta_{i-p}\right)\left(\left(\theta_{i}-\theta_{i-p}\right)\right. \\
\lambda_{i}^{*}(0)=1+\bar{\theta}_{i}^{(2)} /\left(\theta_{i+p}-\theta_{i}\right)\left(\left(\theta_{i}-\theta_{i-p}\right)\right. \\
\lambda_{i}^{*}(p)=-\bar{\theta}_{i}^{(2)} /\left(\theta_{i+p}-\theta_{i-p}\right)\left(\left(\theta_{i+p}-\theta_{i}\right) .\right.
\end{gathered}
$$

We recall that $\theta_{i}=\frac{1}{m} \sum_{r=0}^{m-1} t_{i-r}$ and

$$
\bar{\theta}_{i}^{(2)}=\frac{1}{m^{2}(m-1)} \sum_{(r, s) \in \mathbb{N}_{m}, r<s}\left(t_{i-r}-t_{i-s}\right)^{2}=\frac{S_{1}}{m^{2}(m-1)}
$$

We first compute

$$
\begin{aligned}
& \theta_{i}-\theta_{i-p}=\frac{1}{m} \sum_{r \in \mathbb{N}_{m}}\left(t_{i-r}-t_{i-p-r}\right)=\frac{1}{m} \sum_{r \in \mathbb{N}_{m}} \sum_{k=0}^{p} h_{i-k-r+1}=S_{2} / m . \\
& \theta_{i+p}-\theta_{i}=\frac{1}{m} \sum_{r \in \mathbb{N}_{m}}\left(t_{i+p-r}-t_{i-r}\right)=\frac{1}{m} \sum_{r \in \mathbb{N}_{m}} \sum_{k=0}^{p} h_{i+k-r+1}=S_{3} / m,
\end{aligned}
$$

The proof being essentially the same for all $p \geq m$ and for all $i \in \mathbb{Z}$, we can restrict our study to the cases $p=m$ and $i=m-1$. In that case, we get

$$
\begin{gathered}
S_{2}=m h_{i-m+1}+\sum_{k=1}^{m-1} k(h+h) \geq S_{2}^{\prime}=h_{1}+2 h_{2}+\ldots+(m-1) h_{m-1}, \\
S_{3}=m h_{i-m+1}+\sum_{k=1}^{m-1} k(h+h) \geq S_{3}^{\prime}=(m-1) h_{1}+(m-2) h_{2}+\ldots+2 h_{m-2}+h_{m-1} .
\end{gathered}
$$

Denoting, for $1 \leq k \leq m-1$

$$
s_{k}=h_{1}+\ldots+h_{k},
$$

and $s=s_{m-1}$, we get

$S_{2}^{\prime}=s_{1}+s_{2}+\ldots+s_{m-1}, \quad S_{3}^{\prime}=s+\left(s-s_{1}\right)+\left(s-s_{2}\right)+\ldots\left(s-s_{m-2}\right)=m s-S_{2}^{\prime}$

whence

$$
S_{2} S_{3} \geq S_{2}^{\prime} S_{3}^{\prime}=m s\left(s_{1}+s_{2}+\ldots+s_{m-1}\right)-\left(s_{1}+s_{2}+\ldots+s_{m-1}\right)^{2}
$$


Now, we come back to $S_{1}$ and we shall prove that $S_{1} \leq S_{2}^{\prime} S_{3}^{\prime} \leq S_{2} S_{3}$. $S_{1}$ can be written under the form

$$
S_{1}=\sum_{j=1}^{m-1} \sum h_{i-r+j}=\sum_{i=1}^{m-1} s_{i}^{2}+\sum_{j=1}^{m-1} \sum_{i=j+1}^{m}\left(s_{i}-s_{j}\right)^{2},
$$

from which we deduce

$$
S_{1}=(m-1) \sum_{i=1}^{m-1} s_{i}^{2}-2 \sum_{j=1}^{m-1} s_{j} \sum_{i=j+1}^{m} s_{i}
$$

Moreover, for all $1 \leq i \leq m-1$, we have

$$
(m-1) s_{i}^{2}=m s_{i}^{2}-s_{i}^{2} \leq m s_{i} s_{m-1}-s_{i}^{2}
$$

therefore, we obtain the result

$$
S_{1} \leq(m-1) \sum_{i=1}^{m-1} s_{i}^{2} \leq m s_{m-1} \sum_{i=1}^{m-1} s_{i}-\sum_{i=1}^{m-1} s_{i}^{2}=S_{2}^{\prime} S_{3}^{\prime} \leq S_{2} S_{3} .
$$

Finally, for all $i \in \mathbb{Z}$, we have

$$
\nu_{i}^{*}=1+\frac{2}{m-1} \frac{S_{1}}{S_{2} S_{3}} \leq 1+\frac{2}{m-1}=\frac{m+1}{m-1},
$$

whence $\left\|Q_{p, 2}^{*}\right\|_{\infty} \leq \max _{i \in \mathbb{Z}} \nu_{i}^{*} \leq \frac{m+1}{m-1}$.

In the next section, we prove that the QIs $Q_{p, 2}^{*}$ are near-best in the sense of section 6 .

\section{§. A family of near-best spline discrete QIs}

For dQIs $Q_{p, 2}$ depending on $p \geq m$ parameters, the coefficients (see proof of theorem 5) are given by

$$
\begin{gathered}
\lambda_{i}^{*}(-p)=-\bar{\theta}_{i}^{(2)} /\left(\theta_{i+p}-\theta_{i-p}\right)\left(\left(\theta_{i}-\theta_{i-p}\right)\right. \\
\lambda_{i}^{*}(0)=1+\bar{\theta}_{i}^{(2)} /\left(\theta_{i+p}-\theta_{i}\right)\left(\left(\theta_{i}-\theta_{i-p}\right)\right. \\
\lambda_{i}^{*}(p)=-\bar{\theta}_{i}^{(2)} /\left(\theta_{i+p}-\theta_{i-p}\right)\left(\left(\theta_{i+p}-\theta_{i}\right) .\right.
\end{gathered}
$$

Now, let us write the minimization problem of section 7 in Watson's form. Denote

$$
\tilde{\lambda}_{i}=\left(\lambda_{i}(-p+1), \ldots, \lambda_{i}(-1), \lambda_{i}(1), \ldots, \operatorname{lambda}_{i}(p-1)\right)^{T} \in \mathbb{R}^{2 p-2}
$$


Univ Spline QI/non-uniform partitions

$$
\lambda_{i}^{*}=\left(\lambda_{i}^{*}(-p), 0, \ldots, \lambda_{i}^{*}(0), 0, \ldots, \lambda_{i}^{*}(p)\right)^{T} \in \mathbb{R}^{2 p+1}
$$

Let $A_{i} \in \mathbb{R}^{(2 p+1) \times(2 p-1)}$ be the matrix with the following coefficients (notations of section 7 )

$$
\begin{aligned}
& \text { For } r \in K_{1}: A_{i}(-p, r)=\alpha_{r}, A_{i}(0, r)=\beta_{r}, A_{i}(p, r)=-\gamma_{r}, \\
& \text { For } s \in K_{2}: A_{i}(-p, s)=-\alpha_{s}, A_{i}(0, s)=\beta_{s}, A_{i}(p, s)=\gamma_{s},
\end{aligned}
$$

For $r \in K_{1}: A_{i}\left(r, r^{\prime}\right)=0, r^{\prime} \neq r, A_{i}(r, r)=-1, \quad A_{i}(r, s)=0, s \in K_{2}$,

For $s \in K_{2}: A_{i}(s, r)=0, r \in K_{1}, \quad A_{i}(s, s)=-1, A_{i}\left(s, s^{\prime}\right)=0, s^{\prime} \neq s$.

Then, using these notations, we can write

$$
\left\|\lambda_{i}\right\|_{1}=\left\|\lambda_{i}^{*}-A_{i} \tilde{\lambda}_{i}\right\|_{1}
$$

Theorem 6. Assume that the sequence of knots $T$ satisfies, for all $i \in \mathbb{Z}$, the following properties

$$
\theta_{i-1}+\theta_{i} \leq \theta_{i-p}+\theta_{i+p} \leq \theta_{i}+\theta_{i+1},
$$

then, for all $i \in \mathbb{Z}, \lambda_{i}^{*}$ is an optimal solution of the local minimization problem min $\left\|\lambda_{i}\right\|_{1}$. Thus, for all $p \geq m$, the spline $d Q I s Q_{p, 2}^{*}$ are near-best and their infinite norms are uniformly bounded by $\frac{m+1}{m-1}$. This bound is independent of $p$ and of the sequence of knots $T$.

Proof: According to Watson's theorem, we must find a vector $v^{*} \in \mathbb{R}^{2 p+1}$ satisfying

$$
\left\|v^{*}\right\|_{\infty} \leq 1, \quad A_{i}^{T} v^{*}=0, \quad v^{*}(r)=\operatorname{sgn}\left(\lambda_{i}^{*}(r)\right) \text { for } r=-p, 0, p .
$$

Let us choose

$$
\begin{aligned}
& v^{*}(-p)=-1, v^{*}(0)=1, v^{*}(p)=-1, \\
& v^{*}(r)=-\alpha_{r}+\beta_{r}+\gamma_{r}, \quad \text { for } r \in K_{1}, \\
& v^{*}(s)=-\alpha_{s}+\beta_{s}+\gamma_{s}, \quad \text { for } s \in K_{2} .
\end{aligned}
$$

Then it is easy to verify that the equations $A_{i}^{T} v^{*}=0$ are satisfied. Moreover, the above expressions of $\lambda_{i}^{*}(r)$ for $r=-p, 0, p$ with $\bar{\theta}_{i}^{(2)}>0$ imply that $\operatorname{sgn}\left(v^{*}(r)\right)=\operatorname{sgn}\left(\lambda_{i}^{*}(r)\right)$ for $r=-p, 0, p$. It only remains to prove that, for $(r, s) \in K_{1} \times K_{2}$

$$
\left|v^{*}(r)\right|=\left|-\alpha_{r}+\beta_{r}+\gamma_{r}\right| \leq 1, \quad\left|v^{*}(s)\right|=\left|\alpha_{s}+\beta_{s}-\gamma_{s}\right| \leq 1 .
$$


As $\beta_{r}=1-\alpha_{r}+\gamma_{r}$ for $r \in K_{1}$ and $\beta_{s}=1+\alpha_{s}-\gamma_{s}$ for $s \in K_{2}$, it is equivalent to prove

$$
0 \leq \alpha_{r}-\gamma_{r} \leq 1, \quad 0 \leq \gamma_{s}-\alpha_{s} \leq 1, \quad \text { for }(r, s) \in K_{1} \times K_{2}
$$

We only detail the proof for $r \in K_{1}$, that for $s \in K_{2}$ being quite similar. Using the Vandermonde determinants, we get

$$
\alpha_{r}-\gamma_{r}=V_{i}^{-1}\left(\theta_{i}-\theta_{r}\right)\left(\theta_{i+p}-\theta_{i-p}\right)\left[\left(\theta_{i+p}+\theta_{i-p}\right)-\left(\theta_{r}+\theta_{i}\right)\right],
$$

As $\theta_{i}-\theta_{r} \geq 0$ and $\theta_{i+p}-\theta_{i-p} \geq 0$, we shall have $\alpha_{r}-\gamma_{r} \geq 0$ if and only if

$$
\theta_{r}+\theta_{i} \leq \theta_{i+p}+\theta_{i-p}
$$

for all $r \in K_{1}$. However, since we have $\theta_{r}+\theta_{i} \leq \theta_{i-1}+\theta_{i}$, there only remains the unique condition

$$
\theta_{i-1}+\theta_{i} \leq \theta_{i-p}+\theta_{i+p}
$$

The other inequality $\alpha_{r}-\gamma_{r} \leq 1$ can be written

$$
\left(\theta_{i}-\theta_{r}\right)\left[\left(\theta_{i+p}+\theta_{i-p}\right)-\left(\theta_{r}+\theta_{i}\right)\right] \leq\left(\theta_{i}-\theta_{i-p}\right)\left(\theta_{i+p}-\theta_{i}\right)
$$

Setting $\delta_{1}=\theta_{r}-\theta_{i-p}, \delta_{2}=\theta_{i}-\theta_{r}$, and $\delta_{3}=\theta_{i+p}-\theta_{i}$, the latter inequality can be written

$$
\delta_{2}\left(\delta_{3}-\delta_{1}\right) \leq \delta_{3}\left(\delta_{2}+\delta_{1}\right), \text { or } \delta_{1}\left(\delta_{2}+\delta_{3}\right) \geq 0
$$

which is obviously satisfied. For $s \in K_{2}$, the inequalities $0 \leq \gamma_{s}-\alpha_{s} \leq 1$ are satisfied if and only if

$$
\theta_{i-p}+\theta_{i+p} \leq \theta_{i}+\theta_{i+1}
$$

whence the conditions on the sequence of knots.

Remark. Theorem 6 imposes some conditions on the sequence of knots. For quadratic splines, we have studied arithmetic and geometric sequences: in both cases, the higher is $p$, the stronger are the conditions and for $p \rightarrow+\infty, T$ is closer and closer to a uniform sequence.

**********************************************************

\section{$\S 9$. Some applications}




\subsection{Approximation of functions}

When a spline dQI $Q$ is uniformly bounded independently of the partition, we can apply a classical result in approximation theory (see [5], Th 22, and [11], chapters 2 and 5):

$$
\|Q f-f\|_{\infty} \leq\left(1+\|Q\|_{\infty}\right) d_{\infty}(f, \mathcal{S})
$$

where $\mathcal{S}$ is the space of splines. In particular, when $Q$ is exact on the space $\mathbb{P}_{m}$, then for $f \in C^{m+1}(I)$, one has

$$
\|Q f-f\|_{\infty} \leq C h^{m+1}\left\|f^{m+1}\right\|_{\infty}
$$

for some constant $C$ which does not depend on the given partition. Therefore spline dQIs give the best possible approximation order. More detailed results on error bounds are given in [1],[13] and [23].

\subsection{Quadrature formulas}

Approximating $\int_{I} f$ by $\int_{I} Q_{2}^{*}(f)$, where $Q_{2}^{*}(f)$ is the quadratic spline dQI of section 8 , gives rise to an interesting quadrature formula

$$
\int_{I} Q_{2}^{*}(f)=f_{0} \int_{I} B_{0}+\sum_{i=1}^{n} \mu_{i}(f) \int_{I} B_{i}+f_{n+1} \int_{I} B_{n+1}
$$

As it is well known, $\int_{I} B_{0}=\frac{h_{1}}{3}, \int_{I} B_{n+1}=\frac{h_{n}}{3}$ and $\int_{I} B_{i}=\frac{h_{i-1}+h_{i}+h_{i+1}}{3}$ for $1 \leq i \leq n$. This formula is exact on $\mathbb{P}_{2}$, but in the case of a uniform partition,(see [21]), it is exact on $\mathbb{P}_{3}$ and provides an interesting complementary formula to Simpson's rule in the sense that, in general, errors for both formulas have opposite signs. This will be detailed in another paper, together with applications to integral equations.

\subsection{Pseudo-spectral methods}

One can approximate the first derivatives of a given function $f$ at the data sites

$$
\Theta_{n}=\left\{\theta_{0}=t_{0}, \quad \theta_{i}=\frac{1}{2}\left(t_{i-1}+t_{i}\right), \quad \text { for } 1 \leq i \leq n, \quad \theta_{n+1}=t_{n}\right\} .
$$

by the derivatives of the quadratic spline dQI of section 8.1

$$
Q_{2}^{*} f=f\left(t_{0}\right) B_{0}+\sum_{i=1}^{n} \mu_{i}(f) B_{i}+f\left(t_{n}\right) B_{n+1} .
$$

For interior points $\theta_{i}, 3 \leq i \leq n-2$, we obtain the general formula

$$
\left(Q_{2}^{*} f\right)^{\prime}\left(\theta_{i}\right)=\mu_{i-1}(f) B_{i-1}^{\prime}\left(\theta_{i}\right)+\mu_{i}(f) B_{i}^{\prime}\left(\theta_{i}\right)+\mu_{i+1}(f) B_{i+1}^{\prime}\left(\theta_{i}\right)
$$


which can also be written, by setting $f_{j}=f\left(\theta_{j}\right)$ :

$$
\begin{gathered}
\left(Q_{2}^{*} f\right)^{\prime}\left(\theta_{i}\right)=\frac{1}{h_{i}}\left\{-\sigma_{i} a_{i-1} f_{i-2}+\left[-\sigma_{i} b_{i-1}+\left(\sigma_{i}-\sigma_{i+1}^{\prime}\right) a_{i}\right] f_{i-1}\right. \\
\left.\left[-\sigma_{i} c_{i-1}+\left(\sigma_{i}-\sigma_{i+1}^{\prime}\right) b_{i}+a_{i+1}\right] f_{i}+\left[\left(\sigma_{i}-\sigma_{i+1}^{\prime}\right) c_{i}+b_{i+1}\right] f_{i+1}+\sigma_{i+1}^{\prime} c_{i+1} f_{i+2}\right\}
\end{gathered}
$$

For the first indices $0 \leq i \leq 2$, the coefficients are modified according to the convention $h_{0}=0$, which gives $\sigma_{0}=0, \sigma_{0}^{\prime}=1, \sigma_{1}=1$ and $\sigma_{1}^{\prime}=0$. We thus obtain

$$
\begin{gathered}
\left(Q_{2}^{*} f\right)^{\prime}\left(\theta_{0}\right)=\frac{2}{h_{1}}\left\{\left(a_{1}-1\right) f_{0}+b_{1} f_{1}+c_{1} f_{2}\right\} \\
\left(Q_{2}^{*} f\right)^{\prime}\left(\theta_{1}\right)=\frac{1}{h_{1}}\left\{\left(\sigma_{2} a_{1}-1\right) f_{0}+\left[\sigma_{2} b_{1}+\sigma_{2}^{\prime} a_{2}\right] f_{1}+\right. \\
\left.\left[\sigma_{2} c_{1}+\sigma_{2}^{\prime} b_{2}\right] f_{2}+\sigma_{2}^{\prime} c_{2} f_{3}\right\} \\
\left(Q_{2}^{*} f\right)^{\prime}\left(\theta_{2}\right)=\frac{1}{h_{2}}\left\{-\sigma_{2} a_{1} f_{0}+\left[-\sigma_{2} b_{1}+\left(\sigma_{2}-s i g m a_{3}^{\prime}\right) a_{2}\right] f_{1}+\left[-\sigma_{2} c_{1}+\left(\sigma_{2}-\sigma_{3}^{\prime}\right) b_{2}+\sigma_{3}^{\prime} a_{3}\right] f_{2}\right. \\
\left.+\left[\left(\sigma_{2}-\sigma_{3}^{\prime}\right) c_{2}+\sigma_{3}^{\prime} b_{3}\right] f_{3}+\sigma_{3}^{\prime} c_{3} f_{4}\right\}
\end{gathered}
$$

In the same way, for the last indices $n-1 \leq i \leq n+1$, the coefficients are modified according to the convention $h_{n+1}=0$ and we obtain similar formulas for $\left(Q_{2}^{*} f\right)^{\prime}\left(\theta_{n-1}\right),\left(Q_{2}^{*} f\right)^{\prime}\left(\theta_{n}\right)$ and $\left(Q_{2}^{*} f\right)^{\prime}\left(\theta_{n+1}\right)$. In the case of a unifom partition, the formulas are given in [21]. Concerning error estimates, it is rather easy to verify that $\left(Q_{2}^{*} f\right)^{\prime}\left(\theta_{i}\right)-f^{\prime}\left(\theta_{i}\right)=O\left(h^{2}\right)$ where $h=\max _{1 \leq i \leq n} h_{i}$. A more detailed study will be done elsewhere. These results can be used in pseudo-spectral methods, as described for example in [12] and [29].

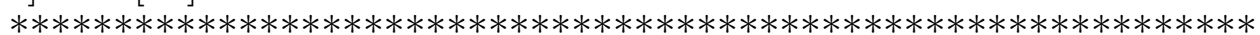

\section{References}

1. D. Barrera, M.J. Ibanẽz, P. Sablonnière: Near-best discrete quasiinterpolants on uniform and nonuniform partitions. In Curve and Surface Fitting, Saint-Malo 2002, A. Cohen, J.L. Merrien and L.L. Schumaker (eds), Nashboro Press, Brentwood (2003), 31-40.

2. D. Barrera, M.J. Ibanẽz, P. Sablonnière, D. Sbibih: Near-minimally normed univariate spline quasi-interpolants on uniform partitions. Prépublication IRMAR 04-12, Université de Rennes, March 2004.

3. D. Barrera, M.J. Ibanẽz, P. Sablonnière, D. Sbibih: Near-best quasiinterpolants associated with H-splines on a three-direction mesh. Prépublication IRMAR 04-14, Université de Rennes, March 2004. 
4. B.D. Bojanov, H.A. Hakopian, A.A. Sahakian: Spline functions and multivariate interpolation, Kluwer, Dordrecht 1993.

5. C. de Boor: A practical guide to splines, Springer-Verlag, New-York 2001. (revised edition).

6. C. de Boor: Splines as linear combinations of B-splines, a survey. In: Approximation Theory II, G.G. Lorentz et al. (eds), 1-47, Academic Press, New-York 1976.

7. C. de Boor, G. Fix: Spline approximation by quasi-interpolants. J. Approx. Theory $\mathbf{8}$ (1973), 19-45.

8. C. de Boor, K. Höllig, S. Riemenschneider: Box-splines, SpringerVerlag, New-York 1993.

9. G. Chen, C.K. Chui, M.J. Lai: Construction of real-time spline quasiinterpolation schemes, Approx. Theory Appl.4 (1988), 61-75.

10. C.K. Chui: Multivariate splines, CBMS-NSF Regional Conference Series in Applied Mathematics, vol. 54, SIAM, Philadelphia 1988.

11. R.A. DeVore, G.G. Lorentz: Constructive approximation, SpringerVerlag, Berlin 1993.

12. B. Fornberg: A practical guide to pseudospectral methods, Cambridge University Press 1996.

13. M.J. Ibañez-Pérez: Cuasi-interpolantes spline discretos con norma casi minima : teoria y aplicaciones. Tesis doctoral, Universidad de Granada, 2003.

14. W.J. Kammerer, G.W. Reddien, R.S. Varga: Quadratic interpolatory splines. Numer. Math. 22 (1974), 241-259

15. B.G. Lee, T. Lyche, L.L. Schumaker: Some examples of quasi-interpolants constructed from local spline projectors. In Mathematical methods for curves and surfaces: Oslo 2000, T. Lyche and L.L. Schumaker (eds), Vanderbilt University Press, Nashville (2001), 243-252.

16. T. Lyche, L.L. Schumaker: Local spline approximation methods, J. Approx. Theory 15 (1975), 294-325.

17. J.M. Marsden, I.J. Schoenberg: An identity for spline functions with applications to variation diminishing spline approximation. J. Approx. Theory 3 (1970), 7-49.

18. J.M. Marsden: Operator norm bounds and error bounds for quadratic spline interpolation, In: Approximation Theory, Banach Center Publications, vol. 4 (1979), 159-175.

19. G. Nürnberger: Approximation by spline function, Springer-Verlag, Berlin 1989.

20. M.J.D. Powell: Approximation theory and methods. Cambridge University Press, 1981. 
21. P. Sablonnière: Bases de Bernstein et approximants splines. Thèse de doctorat, Université de Lille, 1982.

22. P. Sablonnière: On some multivariate quadratic spline quasi-interpolants on bounded domains. In Modern developments in multivariate approximation, W. Haussmann, K. Jetter, M. Reimer, J. Stöckler (eds), ISNM Vol. 145, Birkhäuser-Verlag, Basel (2003), 263-278.

23. P. Sablonnière: Quadratic spline quasi-interpolants on bounded domains of $\mathbb{R}^{d}, d=1,2,3$. Spline and radial functions, Rend. Sem. Univ. Pol. Torino, Vol. 61 (2003), 61-78.

24. P. Sablonnière: Recent progress on univariate and multivariate polynomial or spline quasi-interpolants. Submitted to Proc. IBoMAT 2004, Bommerholz, Germany (February 16-20, 2004). Prépublication IRMAR, Université de Rennes, March 2004.

25. P. Sablonnière: Near-best univariate spline integral quasi-interpolants on non-uniform partitions. Prépublication IRMAR, Université de Rennes, 2004 (in preparation).

26. I.J. Schoenberg: Cardinal spline interpolation, CBMS-NSF Regional Conference Series in Applied Mathematics, vol. 12, SIAM, Philadelphia 1973.

27. I.J. Schoenberg: Selected papers, Volumes 1 and 2, edited by C. de Boor. Birkhäuser-Verlag, Boston 1988.

28. L.L. Schumaker: Spline functions: basic theory, John Wiley \& Sons, New-York 1981.

29. L. N. Trefethen: Spectral methods in Matlab, SIAM, Philadelphia, 2000 .

30. G.G. Watson: Approximation theory and numerical methods, John Wiley and Sons, New-York, 1980.

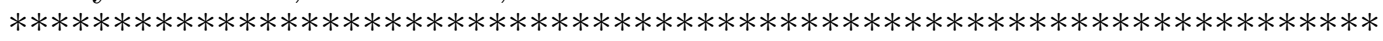

D. Barrera, M.J. Ibañez,

Departamento de Matemática Aplicada,

Facultad de Ciencias, Universidad de Granada,

Campus de Fuentenueva,

18071 GRANADA, Spain.

dbarrera@ugr.es, mibanez@ugr.es

P. Sablonnière, INSA de Rennes,

20 Avenue des Buttes de Coësmes,

CS 14315, 35043 RENNES Cedex, France.

psablonn@insa-rennes.fr

D. Sbibih,

Département de Mathématiques et Informatique, 
Faculté des Sciences, Université Mohammed 1er, 40000 OUJDA, Marocco.

sbibih@sciences.univ-oujda.ac.ma 